\title{
A New System for Monitoring and Analysis of the Knitting Process
}

\author{
Catarino A. \\ Textile Engineering Department \\ University of Minho, Portugal \\ whiteman@det.uminho.pt amrocha@det.uminho.pt
}

\author{
Monteiro J.L. \\ Soares F. \\ Industrial Electronics Department \\ University of Minho, Portugal \\ joao.monteiro@dei.uminho.pt_fsoares@dei.uminho.pt
}

\begin{abstract}
Production monitoring is an important task for proper production planning. For this task the information that a system of this nature can possibly gather assumes significant importance. Among all the information available during production, the detection of faults assumes a crucial role since it directly affects the quality and productivity. This paper presents a system which was developed with the purpose of performing the analysis in real time of the knitting process, supplying the parameters of major concern for production and furthermore, allowing the detection, identification and location of faults.
\end{abstract}

\section{Introduction}

The monitoring of the production of industrial knitting machine is crucial for order planning and scheduling [1]. With this purpose, several solutions are presently proposed by industry manufacturers which provide a very complete and valuable set of information. The systems can supply information concerning standstill periods, the production in meters of the knitted fabric, etc. The trend is to offer the most complete set of information for the technician and management team.

The particular case of fault generation during the production of knitwear has an important impact, since if it is not detected, defective product will be produced and several meters of production can be ruined. Moreover, the machine needs to be stopped and repaired, which can be very time consuming. This is particularly true when the fault is due to a damaged knitting element such as a needle. In this case, the change of the entire set of needles is mandatory, since it is very difficult to detect the damaged element [16]. This change is very expensive both in time and material, since an industrial knitting machine can have several hundreds of needles. The physical reasons that explain the appearance of faults have been documented, and are related with the production nature of knitwear: the constant impact of the knitting elements between them, and the contact with raw material [16]. In fact, the impact on the needles butt can induce vibrations strong enough to break the needle
$[2,3,4,16,17,20]$. On the other hand, the constant contact between the needle and the yarn will produce frictional forces that will weaken the needle structure, thus incurring in an early ageing of the needle and consequent damaging [20]. Nevertheless, the improvements were significant and the lifetime of a presently manufactured needle is significantly higher than their predecessors [20]. However, during intensive use there will be a time that even the most modern knitting element will fail and thus a fault will appear. To prevent this kind of problem, periodic change of the entire set of needles is performed $[16,17]$. This procedure can provide more confidence in avoiding faults, but it does not guarantee fault-free production.

So, several devices were developed in order to detect faults during production. There exist three possible approaches for detecting faults: inspecting the knitting elements, the knitted fabric, or the raw material [16]. The solutions proposed up to now by industry mainly use the first two approaches. Closed latch detectors are an example of the first approach. This is a very specific fault and it does not allow the detection of any other kind of fault. Other technique involves capacitive sensors and pattern recognition in order to detect damaged needles $[5,7,8]$. For the second approach, one device based on optic sensing is mainly adopted, and again pattern recognition techniques are used [5,9]. Is common to observe the two solutions combined in industrial machines. The third approach is however less explored; the most primitive sensing device is the yarn break detector, whose functioning is based on gravity principle [10]. Even this sensor tends to be replaced by optical sensors, with no contact with the yarn. The technology used for fault detection although effective is very case specific, does not detect all the faults and there is no integration or value added information. Research made exploring the third approach proved that is possible to detect faults by inspecting the raw material, in particular the yarn input tension - YIT [11-16]. This is a very important parameter in weft knitting since it is used to tune the feeding of the yarn into the knitting zone. Exceeding YIT will result in yarn break and machine stop. $Y I T$ is indeed a valuable resource of information concerning in particular the knitting process, and in a 
more general term, the overall behavior of the knitting machine, since YIT directly reflects the influence of the different mechanisms involved in the production of the knitted fabric [16]. It was observed that this approach allows the detection of the same and some more faults that the previously mentioned sensors do, each one separately. Also, the shape of the faults are different which suggest the use of automatic recognition of the fault nature[14]. These facts can be an excellent reason for an investment on this approach for future knitting machines.

\section{The Measuring System}

Knitlab is a hardware and software based solution which, when assembled on a knitting machine, allows the monitoring and analysis of the knitting process. The method used for the analysis of the knitting process is based on a crucial parameter for weft knitting production - yarn input tension (YIT). The parameter reflects with high accuracy what happens during the formation of a loop, as previous research has proved $[4,16]$. If some kind of problem occurs, it will be reflected in the YIT variation, which in his turn can be further analyzed and interpreted as being a random behavior or an extraordinary event classified as a fault.

The system is based on force, optical and positioning sensors. The force sensors allow the measurement of the $Y I T$, and the position sensors are responsible of supplying the positioning, speed and other important parameters for knitting production. Finally, the optical sensor is used for synchronizing purposes. The system was designed to be easily assembled on a knitting machine, without the need for any kind of structural modification. Figure 1 illustrates the way the sensors are assembled.

KnitLab allows the measurement of virtually any kind of yarn currently used on weft circular knitting machines, since it measures forces from ranges $0.0-5.0 \mathrm{cN}$ up to 0.0 $200.0 \mathrm{cN}$. The sensor device is based on strain gauges and is capable of measuring the YIT at industrial top speeds, which are about 1.0 to $1.5 \mathrm{~m} / \mathrm{s}$. These speeds represent more than 2000 needles per second.

Calibration of the force sensor is available and also static and dynamic characterization, as figure 2 illustrates. The signal is filtered before the sampling operation performed by the data acquisition board and the cut-off frequency is determined automatically by the equivalent number of needles per second. All adjustments can be made through software programming, in manual or automatic mode.

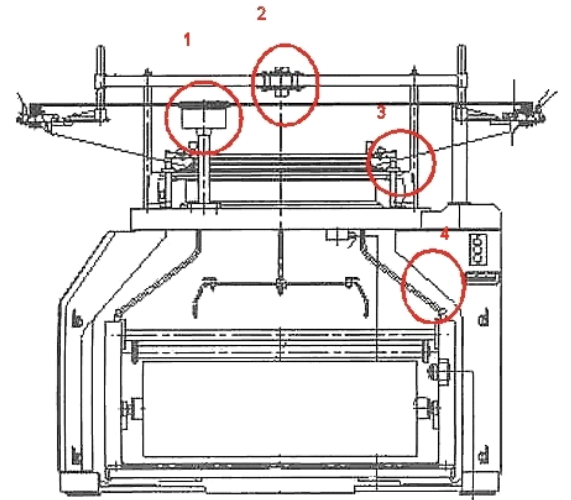

Figure 1. Sensor's location on the knitting machine.

KnitLab supports two different acquisition rate methods, although it preferably uses the equally spaced interval samples. Through this method, one can obtain a more accurate location of faults, in case of their appearance, since constant time rate need further processing due to speed fluctuations. For the purpose of producing equally spaced sampling rate, incremental encoders were used, while for time rate the internal data acquisition board counters are used.

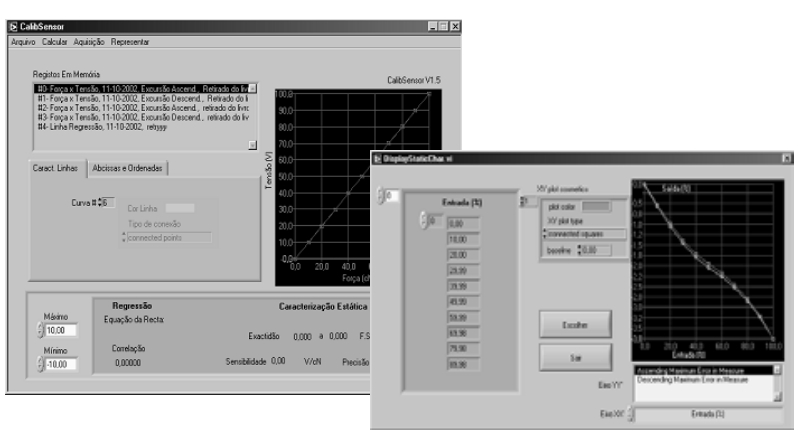

Figure 2. Characterization and calibration of the force sensor. Regression curve (left) and maximum error in measurement (right).

Two different sources for equally spaced interval can be selected: from the encoder located on the main cylinder or from the encoder located on one of the yarn feeding systems. The first option is used whenever is possible, since it prevents possible sliding and assures a fixed number of points per cylinder revolution.

In the case of unavailability of the needles cylinder, one can use the rotating movement of one yarn feeding system for the generation of the sampling rate. The feeder rotating movement is extracted directly from the main cylinder movement (figure 3 ). Thus, a multiple number of points will be produced per cylinder rotation. In the case of having more points than intended to, the application will reorganize the acquired waveform to the desired number of points, by using interpolation or cubic splines. This encoder as also another role, as it allows the 
measurement of the yarn consumption and speed. At the present moment, the error concerning the yarn consumption is about $1 / 500$ th of the feeder perimeter, which can represent for a typical industrial feeder less than $3.0 \times 10-4 \mathrm{~m}$.

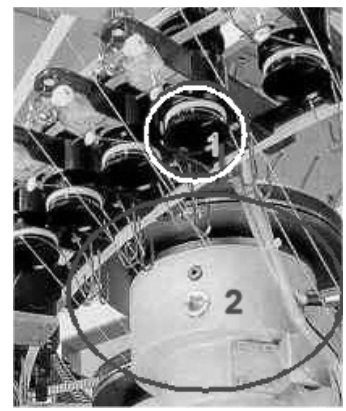

Figure 3. Detail of one yarn feeder (1) and the shaft

(2) which is connected to the needles cylinder.

This set of sensors and conditioning board allows the determination and monitoring of the following parameters:

- $\quad$ Yarn Input Tension (YIT);

- Knitting machine's speed (linear expressed in $\mathrm{m} / \mathrm{s}$, and circular expressed in $\mathrm{rot} / \mathrm{min}$ );

- $\quad$ Yarn Speed (m/min);

- Yarn consumption per cylinder revolution;

- $\quad$ Tightness factor $(\mathrm{K})$;

- $\quad$ Loop length.

The application was developed under LabVIEW environment and can be generally described by the scheme represented in figure 4. KnitLab allows the acquisition of the data, its storage in proprietary structured records and retrieval. In order to analyze the data in other mathematical packages is possible to export/import in spreadsheet format. Is also possible to directly export in spreadsheet format the data previously acquired. There is a set of tools that allow the evaluation of the measuring system correct functioning. This includes all sensors as well as the conditioning board. The general procedure for performing an analysis on the YIT is as follows: The sensor is calibrated according to a previously made characterization procedure. Then, the measuring system is programmed by introducing the information comprehending the knitting machine's parameters and the yarn characteristics. This includes the cylinder diameter, number of needles, cams, feeders, the yarn title and nature, among other parameters. The latter in particular will determine and suggest a configuration of the measuring system. At the same time, this information is gathered on a record that will also contain the waveform itself. The user can then load previously stored records and analyze with the tools available (figure 5), which comprehend frequency analysis, correlation analysis, variance analysis, digital filtering, synchronized time/space average, beyond other tools.

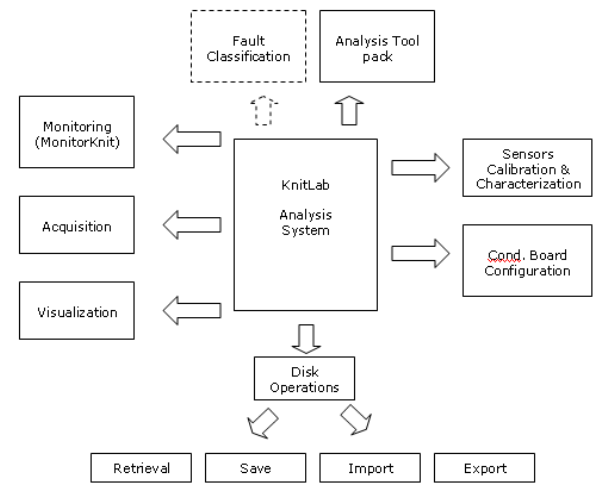

Figure 4. General structure of the Knitlab Analysis system.

Finally, the application can also invoke the monitoring and surveillance system, which is the focus of this paper.

The application is also able to perform a real-time monitoring of the knitting process as well as to detect faults and abnormalities during production. The monitoring module is configured according to the previously recorded parameters of the knitting machine and raw material. As soon as the knitting machine starts working, the YIT tension is acquired, processed and displayed in three different plots: the YIT as is acquired; the waveform after specific digital processing; the waveform summarized in one single value per entire revolution. Since the amount of information can be very significant due to the speed of the knitting machine, only the summarized value of the YIT, speed, and knitting parameters are stored. Also 10 courses are stored in a circular buffer. The rest of the information is discarded.

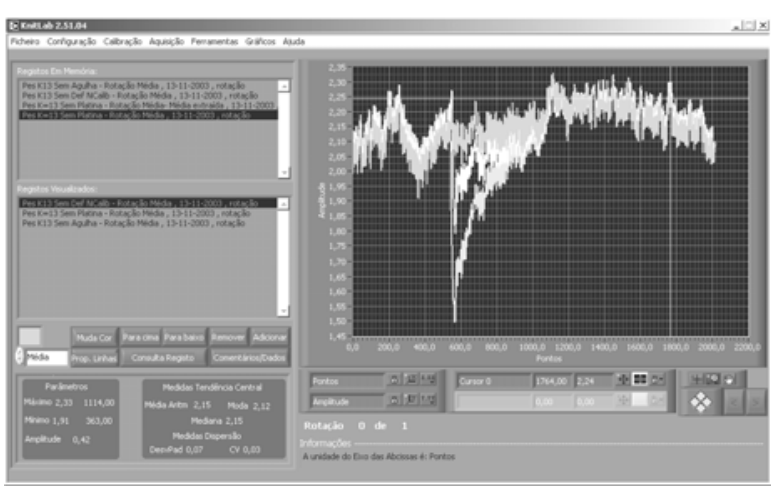

Figure 5. Main view of KnitLab. Three examples are plotted: fault-free, broken needle \& missing sinker.

When the fault detection module is activated, a decision module inspects the waveform and in case of detecting a fault, the system signals the problem to the technician and orders the knitting machine to stop. Meanwhile the system automatically starts recording all 
information until the knitting machine stops. In the next section an overview of the processing methods will be presented.

\section{Monitoring and Fault Detection}

The examples that will be presented here refer only to a particular kind of structure, jersey, and were obtained from a sample knitting machine with different speeds, ranging from $0,15 \mathrm{~m} / \mathrm{s}$ to $0,6 \mathrm{~m} / \mathrm{s}$, for polyester continuous filament yarn 240 dtex and cotton 24 tex yarn. The knitting machine has 168 needles, diameter of 3.75 inches, and positive feeding.

When some abnormality occurs in the knitting process, it will always be reflected in the YIT. The problem that one faces is to distinguish between some random event, which will not produce a defect in the product and the situation that indeed promotes a fault, and thus a defect in the knitted product. In order to expose the problem, let's consider one rotation of the needles cylinder. One rotation represents one course, which means a horizontal row of loops produced by adjacent needles during that knitting cycle [17]. In terms of signal this represents a fairly well shaped sinusoidal waveform, with a frequency equivalent to the time elapsed between each loop formation [11]. This can be easily explained from the geometry of the knitting cams, which are the responsible of the up and down movement of the needle when forming a loop. Thorough research made proposed non-linear cams in order to maximize the speed of the knitting machines and thus the increase of the production $[2,3]$. However, due to the knitting process itself, the yarn feeding systems, eccentricities and the yarn nature and characteristics, the resulting wave-form is somewhat different, as figure 6 illustrates. As it can be seen, there exists an intrinsic variability which is expected to have a random behavior. The synchronized time average (STA) technique [18], allows the removal of this inherent variability and a more clear observation of the knitting process behavior during one course (Figure 6).

Since the variability is supposed to be random, it is expected that the variability for each n-point for all rotations would have a behavior similar to a normal distribution. With the purpose of testing the normality, a qui-square normality test was implemented. The results showed that, in general terms and for a significance of 0.05 , indeed the variability can be assumed to have normal distribution behavior. STA constitutes an excellent technique for detecting permanent problems, since they will appear in every course and thus will not be absorbed. An example is illustrated in figure 6 .

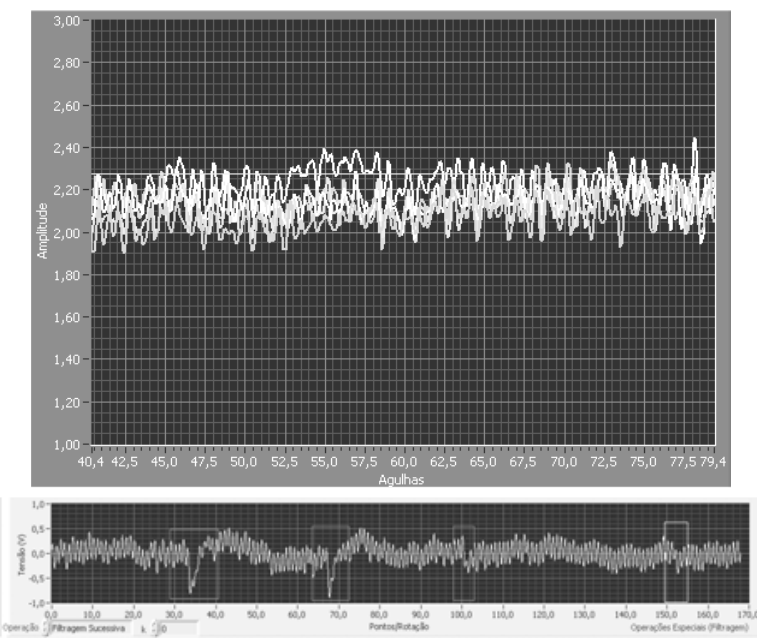

Figure 6. YIT waveforms. Detail of four revolutions (top), and STA applied in real time monitoring with two faults (bottom).

This situation represents several problems detected during production which later were identified as missing needles and a damaged sinker. The problem was confirmed in the knitting machine and after repairing the faulty elements the located abnormalities vanished.

A damaged knitting element produces a decrease on the YIT for a well determined magnitude and time or space interval, and as a consequence a fault in the knitted product, as figure 6 (bottom) illustrates $[4,14,15,16]$. The problem is very well located in the knitting machine cylinder and the application provides the location both in needle position as well as in cam position, which result in significant time savings on repairs. The accuracy and precision are excellent, ranging from one to three needles (depends on the method used for the representation of the YIT waveform). The position of the faulty element can be visually detected but is also determined automatically, by scanning the waveform for a maximum or minimum, depending on the technique used. Different causes for faults produce different shapes, fact that can be used for distinguishing the faults.

So, the detection of one fault seems to be straightforward. However, there is a problem. The intrinsic variability of the YIT during one course can disguise a potential fault and thus pass without being detected. This would result in defective knitted product with an important economic impact. Unfortunately, the STA will not be very useful for this case since it will absorb that abnormality. Another problem is the need to detect the fault as soon as possible, in order to avoid as much as possible the amount of defective product. Some techniques involving specific filtering of the YIT waveform were attempted and all of them are available in the application. One of the techniques involves the 
Matched Filter method [18,19], in which the signal is compared to a template that represents the inverted signal under pursuit. This method is very useful when the signal is buried with noise, as it is in this case. When the waveform matches the template, this measure will return a maximum.

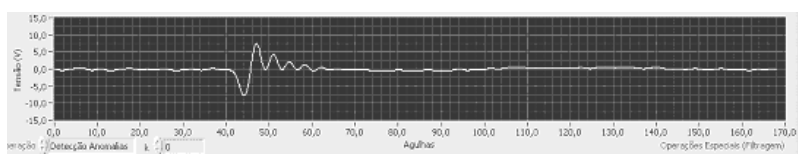

Figure 8. Matched filter applied to YIT for needle break situation.

The experiments showed that this method can be successfully used for detecting a particular fault. However, the draw-backs are that it can detect only one kind of fault, meaning that it would be necessary to have as many templates as the possible fault situations, working in parallel.

The other approach involves the Average Magnitude Cross-Difference (AMCD) [18,19]. In this technique, blocks of pre-processed YIT waveform are compared with a previously selected template with length $\mathrm{N}$. This template is a slice from an arithmetic average of at least 30 YIT fault-free waveform revolutions. Whenever the two signals are similar in shape, the $A M C D$ value becomes a minimum. The template can be directly obtained using the developed application. Figure 9 illustrates the results obtained using this method. The biggest advantage of this method is that it can detect any kind of fault.
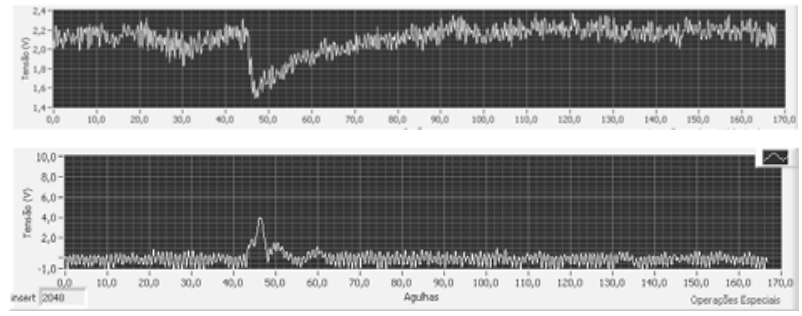

Figure 9. Needle without hook. The picture above shows the YIT as it was acquired, and the second picture shows the processed waveform with AMCD.

This approach allows the detection of an event that occurs once in time as well as the other permanent phenomenon. A threshold exists that was implemented on the decision module in order to detect faults.

The YIT can also be represented as one single value and thus plotted on charts, such as a quality control chart. This representation is more concise than the representation on graphics of the entire YIT for each course. Moreover, the permanent inspection of the YIT waveform can be difficult to observe in industrial environment, since the technician will have several knitting machines under his charge, and the refreshing speed can also be so high that is impracticable to ob-serve the YIT. Instead, it would be very useful to have the average YIT value as well as the knitting process of one entire course represented on a plot.

The monitoring application supplies this functionality by using a measure of how close the presently acquired YIT waveform is when compared with an expected faultfree YIT waveform.

Figure 10 illustrates the application running and the above mentioned comparison measure plotted on a chart.

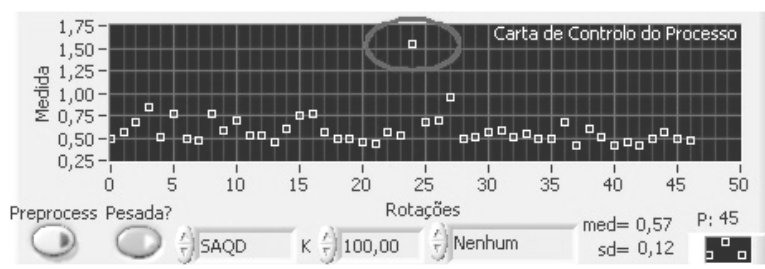

Figure 10. YIT resumed in one single value and plotted on a process chart.

One can easily identify a problem occurring in course number 24. From the magnitude is possible to determine when it happened and identify it on the knitwear and in some cases the cause of the fault. Thus a threshold is evident between fault-free functioning and a fault occurring during production and it is implemented in the application for detecting an abnormality. This method can also be used to count the faults occurred during production. The location of the damaged element can be determined by inspecting the YIT waveform.

The methods presented for detecting faults during production all have their advantages and drawbacks. For instance, $S T A$ is an excellent tool for detecting permanent malfunctions during production, since it absorbs the natural variability produced on the YIT. It allows the location of the cause with the highest accuracy of all methods. However, this method revealed to be totally inefficient for an isolated event, i.e., if one fault occurs once in time.

The methods involving matched filtering, on the contrary, allow the detection of this kind of phenomenon. In particular, $A M C D$ measure gave excellent results. It is possible to locate the origin, with a cost of a slightly inferior accuracy. However, is a little bit more difficult to identify permanent faults, since they can be disguised due to the effect of the template used. Both representations are very useful for locating the fault origin, but are difficult to interpret in on line monitor-ing. The measures that summarize the YIT of one course in one single value can overcome this difficulty and inclusively they allow in some cases to identify the cause. Unfortunately they cannot inform about the location.

So, these three methods should be used together for an effective detection of faults. 


\section{Further Developments}

Further investigation is being performed with the purpose of improving the techniques of fault detection. A database is being collected in order to proceed with the next stage of this application, which is the development of a pattern recognition system for identification of the faults. Some experiments were already made using clustering and discriminating techniques with promising results. It is also intended to extend this application to other knitting structures and machines.

\section{Conclusions}

This paper presented a system developed with the purpose of monitoring and detecting faults during the production of weft circular knitting machines. The system can be assembled in any circular machine and uses the yarn input tension (YIT) as approach to detect faults and monitor the knitting process. It supplies the technician with a significant set of information concerning the major parameters of the knitting production. An overview of the system was presented, with particular emphasis on the methods used for detecting the faults their advantages and drawbacks. The set of tools presented are capable of successfully detect and locate faults in on line production, thus contributing for the reduction of stop and repair times, and thus reducing defective knitwear.

\section{Acknowledgements}

The authors wish to thank the sponsors of this research project, FCT - Fundação para Ciência e Tecnologia, project POSI/SRI/39824/2001 and the University of Minho.

\section{References}

[1] G. Buhler, L. Pestel, H. Hait, "Requirements in regard to future circular knitting assurance systems- Practical trial with a future-oriented monitoring system", Melliand textilberichte, 1 , 1994, p 39-42

[2] Black D. H., Munden D. L., "Increasing the rates of fabric production of weft knitting machinery, Parts I \& II, Journal of Textile Institute, 61, 1970

[3] Lau, T.W.Y., Knapton J.J.F., "The design and dynamics of non-linear cams for use in high speed knitting machines, Parts I to IV, Journal of Textile Institute, 69, 1978

[4] T Push, I. Wunsh, R. Seifert, P. Offermann, "Fine structure of yarn tension on large-diameter circular knitting machines", Melliand textilberichte 1-2, 1997, p 52-55
[5] H. Behr, "Accessories for circular and flat knitting machines”, Knitting Technology, 1996, Vol. 18, 4, p. 184-189

[6] J. Bauer, "Positive yarn feed on knitting machines", Knitting Technol-ogy, 1996, Vol 18, 4, p. 190-193

[7] Needle Detector NW from Memminger-IRO, reference 335.905.001.07 DSNL/5, official website http://www.memminger-iro.de/

[8] Digital Needle Sensor Type 4020, Protechna, official website http://www.protechna.de/e/nadtast_dd.htm

[9] Fabric Scanner LMW 3, Memminger-IRO, reference 040.905.000.01 DUDD/40, official website http://www.memminger-iro.de/

[10] Yarn detectors OFW/UFW, Memminger-IRO, official website http://www.memminger-iro.de/

[11] A. P. Catarino, A.M. Rocha, J. L. Monteiro, "Monitoring Systems for fault detection on circular knitting machines through yarn input tension", ICOM 2003 - International Conference on Mechatronics, ISBN 186058420 9, pg 465-470

[12] André Catarino, Ana Rocha, João Monteiro, "Low Cost Sensor for the Measurement of Yarn Input Tension on Knitting Machines", ISIE 2003 - International Symposium on Industrial Electronics, ISBN 0-7803-7912-8

[13] André Catarino, Ana Rocha, João Monteiro, "Monitoring Knitting Process through Yarn Input Tension: New Developments", IECON 2002, ISBN 0-7803-7475-4

[14] A. Catarino, A. M. Rocha, J. Monteiro; "Automatic Fault Identification in Knitting Machines", SPPRA 2001 IASTED International Conference, pp.5-10

[15] M. Araújo, A. Rocha, H. Hong, A. Catarino, "Towards the Automatic Control of Sewing and Knitting Operations", World Automation Congress 2000, pp. 636-641

[16] A. Catarino, Dinâmica da Tricotagem: Estudo da Dinâmica da Tensão de Entrada do Fio e sua Aplicação em Controlo de Qualidade, Msc Thesis, University of Minho, 1998

[17] David J. Spencer, Knitting Technology, 2nd Ed., Pergamon Press: 1989

[18] E. C. Ifeachor and B W. Jervis, Digital Signal Processing a practical approach, 2nd edition, Prentice Hall, Harlow, 2002, pg 877-887

[19] B.U. Kohler, C. Hennig, R. Orglmeister, "The Principles of Software QRS Detection", IEEE Engineering in Medicine and Biology, January/February, 2002, pg 42-57

[20] Groz Beckert technical Info, KN 2, KN 7, KN 9, KN 10 